\title{
OCT is not useful for detection of minimal diabetic retinopathy in type 1 diabetes
}

\author{
Alessandro Ciresi - Marco Calogero Amato - Daniele Morreale • \\ Raffaella Morreale $\cdot$ Fausto Di Giovanna $\cdot$ Sandra Carità · \\ Gaetano Lodato · Aldo Galluzzo · Carla Giordano
}

Received: 13 November 2009/Accepted: 14 April 2010/Published online: 8 May 2010

(C) Springer-Verlag 2010

\begin{abstract}
Optical coherence tomography (OCT) has been proven useful in measuring retinal thickness (RT) in patients with diabetes, although with discordant results in different studies. We examined RT in patients with type 1 diabetes (T1D) with or without minimal diabetic retinopathy (MDR) to test whether OCT is able to identify early retinal changes and potential correlations with metabolic parameters. RT of 102 patients with T1D (53 females, 49 males, aged $27.03 \pm 7.4$ years) and of 42 healthy controls was examined, with analysis of nine different sectors (fovea, four pericentral and four peripheral sectors). According to the results of basal fundus photography, patients were divided into two groups, without MDR (48 cases) and with MDR (54 cases). Patients with proliferative DR or macular edema were excluded. No difference was found between patients with or without MDR and the control group for all OCT parameters investigated. Mean HbA1c of the last 5 years $(P<0.001)$, microalbuminuria $(P=0.002)$, total $(P=0.046)$ and LDL cholesterol $(P=0.007)$ and triglyceride $(P<0.001)$ levels were higher in patients with MDR, along with higher prevalence of hypertension $(P=0.013)$, younger age at diagnosis $(P=0.018)$ and longer diabetes duration $(P<0.001)$ with
\end{abstract}

A. Ciresi · M. C. Amato - A. Galluzzo · C. Giordano ( $\square)$ Section of Endocrinology, Dipartimento di Oncologia Sperimentale e Applicazioni Cliniche (DOSAC), Università degli Studi di Palermo, piazza delle Cliniche 2, 90127 Palermo, Italy

e-mail: cgiordan@unipa.it

D. Morreale $\cdot$ R. Morreale $\cdot$ F. Di Giovanna $\cdot$ S. Carità .

G. Lodato

Section of Ophthalmology, Dipartimento Universitario di Neuroscienze Cliniche (DINEC), Università degli Studi di Palermo, Palermo, Italy regard to the patients without MDR and controls, although no significant correlation between these parameters and RT was found. Our study suggests that MDR without macular edema in patients with T1D cannot be detected with OCT. Therefore, the conventional diagnostic methods are mandatory to detect early DR.

Keywords Retinopathy - Type 1 diabetes · OCT

\section{Introduction}

Diabetes mellitus is commonly associated with both microvascular and macrovascular complications increasing morbidity and reducing life expectancy. There is therefore more and more interest in identifying diagnostic and therapeutic strategies to reduce or delay the possible onset of these complications [1,2]. Diabetic retinopathy (DR), one of the main causes of blindness in adults, is a relatively common complication, often causing no symptoms or only mild vision problems. Recent studies have identified several new potential mechanisms to explain the etiopathogenesis of pericyte loss or proliferative retinopathy, as the link between the overexpression of human angiopoietin-2 and the pericyte loss or genetic factors [3, 4]. The diagnostic strategies for early detection of DR are of high priority to avoid or prevent loss of vision in patients with diabetes. Ophthalmoscopy, fundus photography and, if necessary, fluorescein angiography are the common techniques used to diagnose DR [5, 6]. Recently, a sensitive, non-invasive and high-resolution method for examining ocular tissue, optical coherence tomography (OCT), has been proposed for early detection of DR; this technique is able to detect even very small changes in the retinal layers, therefore improving the diagnosis and management of DR 
[7-9]. In patients with macular edema, it is generally accepted that OCT is a more sensitive technique for measuring retinal thickness (RT) and volume [10-12], while the current gold standard for the identification of the incipient lesions related to subclinical macular edema or without loss in cells of the retinal layer is fundus photography [6]. Several studies have reported OCT-documented alterations in the RT of patients with diabetes with DR, although no unanimous results have been obtained [13-16]. Conversely, only a few authors have published data reporting that OCT is useful in the early stages of DR, as in the presence of subclinical edema $[9,13]$. Both increased and decreased thickness in different retinal sectors have been identified in patients with diabetes without clinically detectable macular edema, and without any correlation between RT and metabolic parameters. The aim of our observational study was to determine the variation in OCTmeasured macular thickness in the eyes of selected patients with type 1 diabetes (T1D) with or without signs of minimal DR, in order to detect possible early retinal changes and to investigate factors that might influence any variation in macular thickness.

\section{Materials and methods}

\section{Patients}

The study involved 102 consecutive patients with type 1 diabetes (mean age $27.03 \pm 7.4$ years), made up of 53 females $(52 \%)$ and 49 males (48\%), observed in our Outpatients Clinic of Diabetology and Endocrinology Unit from October 2006 to March 2007. Patients gave their informed consent according to the guidelines of the Institutional Review Board at the Faculty of Medicine (University of Palermo). All patients were treated with insulin basal-bolus therapy (3 short-acting or analog injections before meals and one long-acting). Clinical and laboratory parameters were available at diagnosis and then every 3 months, from the beginning of the study. Data included physical examination, mean value of glycosylated hemoglobin (HbA1c) during the previous 5 years, lipid profile (total, HDL and LDL cholesterol, triglycerides), renal function with serum and urine creatinine and microalbuminuria and blood pressure. At the moment of recruitment, patients were divided into two groups depending on the results of fundus photography, those without retinal alterations (group A, 48 cases) and those with minimal diabetic retinopathy (MDR) with no evidence of clinically significant macular edema (CSME) (group B, 54 cases). MDR was defined as the presence of at least two microaneurysms and/or minor hemorrhages in the central retina [17]. A healthy control group was included (group C, 42 cases), age and gender matched, with no family history of ocular disease, glaucoma or any relevant systemic disease.

CSME is defined as retinal thickening within $500 \mu \mathrm{m}$ of the center of the macula or, alternatively, hard exudates within $500 \mu \mathrm{m}$ from the center of the macula with thickening of the adjacent retina, in accordance with ETDRS) [18].

\section{OCT measurements}

Quantitative assessment of retinal thickness was performed with Stratus OCT-Model 3000 (Carl Zeiss Meditec, Dublin, CA, USA), using the fast macular thickness OCT scan protocol. Briefly, retinal thickness was calculated in 3 areas: fovea (central circle, with a diameter of $1 \mathrm{~mm}$ ), pericentral area (shaped ring comprised between an inner diameter of $1 \mathrm{~mm}$ and an outer diameter of $3 \mathrm{~mm}$ ) and peripheral or paracentral area (inner diameter of $3 \mathrm{~mm}$ and outer diameter of $6 \mathrm{~mm}$ ). Fast macular thickness scan protocol was performed in both eyes in all subjects and the same expert examiners obtained OCT scans for each eye.

Statistical analysis

Statistical analyses were performed using SPSS for Windows (2001 release 11.0, Chicago, USA). Continuous variables were analyzed as mean values \pm standard deviation (SD). Rates and proportions were calculated for categorical data. For categorical variables, differences were analyzed with $\chi^{2}$ test and Fisher's exact test when appropriate. Differences among continuous variables with normal distribution were analyzed by Student's $T$-test; for continuous variables without normal distribution, we used non-parametric tests and differences were analyzed by the Mann-Whitney $U$-test. Differences among the three groups (control subjects, group A and group B) were analyzed with Univariate ANOVA and Bonferroni post hoc test. Correlations among RT and metabolic parameters were determined by using Pearson's test. $P$ value of $<0.05$ was considered statistically significant.

\section{Results}

Table 1 shows the features of the patients with diabetes subdivided according to the results of the fundus examination at the time of recruitment. There was no significant difference in age and sex between group A and B, whereas in group $\mathrm{B}$ the age at the time of diagnosis of T1D was lower than in group A $(P<0.01)$. Patients with minimal DR (group B) had a higher disease duration compared with group $\mathrm{A}$ as well as higher HbA1c levels $(P<0.001)$. The difference in HbAlc levels was found not only at the time 
Table 1 Features of patients with type 1 diabetes without (NDR) and with minimal diabetic retinopathy (MDR)

\begin{tabular}{|c|c|c|c|}
\hline & $\begin{array}{l}\text { Group A } \\
\text { (NDR) }\end{array}$ & $\begin{array}{l}\text { Group B } \\
\text { (MDR) }\end{array}$ & $\begin{array}{l}P \\
A \text { vs. } B\end{array}$ \\
\hline & No. 48 & No. 54 & \\
\hline Age (years), Mean (SD) & $25.88(7.24)$ & $29.15(7.61)$ & $0.189^{\S}$ \\
\hline Diabetes onset age, Mean (SD) & $19.62(7.64)$ & $13.61(6.58)$ & $0.018 *$ \\
\hline \multicolumn{4}{|l|}{ Gender } \\
\hline M, No. (\%) & $25(52)$ & $24(44.5)$ & \multirow[t]{2}{*}{0.567} \\
\hline F, No. $(\%)$ & $23(48)$ & $30(55.5)$ & \\
\hline Duration of diabetes (years), Mean (SD) & $6.25(5.69)$ & $15.53(3.73)$ & $<0.001^{*}$ \\
\hline HbA1c (at the time of OCT) (\%), Mean (SD) & $7.6(1.6)$ & $10.6(2.65)$ & $<0.001^{\S}$ \\
\hline Mean $\mathrm{HbA} 1 \mathrm{c}$ (of the last 5 years) (\%), Mean (SD) & $7.5(1.22)$ & $9.9(1.4)$ & $<0.001^{*}$ \\
\hline Hypertension, No. (\%) & $2(8.33)$ & $6(46.15)$ & 0.013 \\
\hline Total cholesterol (mmol/l), Mean (SD) & $4.28(0.98)$ & $5.05(0.53)$ & $0.004 *$ \\
\hline HDL cholesterol (mmol/l), Mean (SD) & $1.57(0.47)$ & $1.33(0.40)$ & $0.119 *$ \\
\hline LDL cholesterol (mmol/l), Mean (SD) & $2.24(0.99)$ & $2.96(0.44)$ & $0.003 *$ \\
\hline Triglycerides (mmol/l), Mean (SD) & $1.01(0.40)$ & $1.65(0.33)$ & $<0.001^{*}$ \\
\hline Microalbuminuria (mg/l), Mean (SD) & $4.69(6.49)$ & $127.57(271.37)$ & $0.002^{\S}$ \\
\hline
\end{tabular}

* Student's T-test; ${ }^{\S}$ Mann-Whitney test

of OCT analysis but also during the previous 5 years, as shown in the clinical records (mean values calculated as average of values recorded every trimester). A higher prevalence of hypertension and higher total cholesterol, LDL, triglyceride and microalbuminuria levels were observed in group B in comparison with group A, confirming that group B had less metabolic control. A linear correlation showed that two retinal areas significantly correlated with metabolic parameters; in fact, the thickness of the right peripheral area significantly correlated with HbA1c at the time of the study ( $r=0.424, P=0.009)$ and with HbA1c mean values of the previous 5 years $(r=$ $0.406, P=0.013)$ while the thickness of the right pericentral area correlated with microalbuminuria $(r=0.382$, $P=0.020)$. No correlation was found for other retinal areas. Furthermore, when we compared RT mean values of groups A, B and the age-matched control subjects, no significant difference was detected for any of the parameters examined (Table 2).

\section{Discussion}

Diabetic complications, including diabetic retinopathy, have long been the subject of studies aimed at identifying clear etiopathogenetic factors with diagnostic and therapeutic implications [3, 4]. OCT is currently considered an important biomedical imaging tool for high-resolution retinal evaluation, but without unanimous data in the absence of clinically detectable macular edema [9, 13]. In our study, we investigated whether OCT is able to detect early impairment of diabetic retinal thickness in patients with

Table 2 Retinal thickness (RT) values (expressed as $\mu \mathrm{m}$ ) in patients with diabetes and control subjects

\begin{tabular}{|c|c|c|c|c|c|c|c|c|c|}
\hline & $\begin{array}{l}\text { Group } \\
\text { (NDR) }\end{array}$ & & $\begin{array}{l}\text { Group } \\
\text { (MDR }\end{array}$ & & $\begin{array}{l}\text { Group } \\
\text { (Contr }\end{array}$ & & \multirow[t]{3}{*}{$\begin{array}{l}P^{*} \\
A \text { vs. } B\end{array}$} & \multirow[t]{3}{*}{$\begin{array}{l}P^{*} \\
A \text { vs. } C\end{array}$} & \multirow[t]{3}{*}{$\begin{array}{l}P^{*} \\
B \text { vs. } C\end{array}$} \\
\hline & \multicolumn{2}{|c|}{ No. 48} & \multicolumn{2}{|c|}{ No. 54} & \multicolumn{2}{|c|}{ No. 42} & & & \\
\hline & Mean & SD & Mean & SD & Mean & SD & & & \\
\hline Fovea (right) & 206.5 & 21.3 & 206 & 23.5 & 201.1 & 18.9 & 1 & 1 & 1 \\
\hline Fovea (left) & 206.1 & 19.9 & 207.9 & 26.9 & 199.6 & 18.7 & 1 & 0.770 & 0.702 \\
\hline Pericentral (right) & 267.6 & 13.1 & 274.4 & 26.6 & 271.6 & 15.5 & 0.786 & 1 & 1 \\
\hline Pericentral (left) & 269.7 & 12.7 & 275.2 & 19.6 & 271.8 & 13.5 & 0.823 & 1 & 1 \\
\hline Peripheral (right) & 241.8 & 12.3 & 253 & 22.7 & 240.5 & 13.1 & 0.109 & 1 & 0.046 \\
\hline Peripheral (left) & 242.5 & 15.8 & 243.9 & 16.8 & 241.2 & 13.5 & 1 & 1 & 1 \\
\hline
\end{tabular}

* Bonferroni post hoc test 
T1D with no signs of retinopathy and in patients with minimal retinopathy, without macular edema, in comparison with healthy subjects. Several studies have reported that OCT provides important information about the retina of patients with diabetes. The majority of clinical studies have been performed on heterogeneous groups of patients, with macular edema and/or including patients with type 2 diabetes $[19,20]$. In our opinion, the strength of this study lies is the careful selection of patients, with the exclusive inclusion of patients with type 1 diabetes mellitus and the exclusion of patients with macular edema or proliferative retinopathy. It is in fact generally accepted that OCT is the technique of choice for understanding the anatomy of macular edema [10] but also in the absence of the latter, both increased and decreased thicknesses in different retinal sectors have been shown in different studies. Biallosterski et al. demonstrated significantly decreased RT, but only in pericentral sectors, in patients with T1D with minimal DR compared to healthy controls, assuming a loss of intraretinal neural tissue in the earlier stage of DR, while no difference was detected in the remaining areas [16]. Conversely, Lattanzio et al. showed increased macular thickness in patients with diabetes according to the progression of DR and the presence of macular edema, thus considering OCT a sensitive technique for early detection of diabetic macular abnormalities [20]. In our study, RT in patients with T1D with minimal or no retinopathy is not significantly different from that of the control group. This is consistent with previous reports [21]. Furthermore, OCT-measured RT and its relationship with metabolic factors in patients with T1D have also been assessed. Recently, a correlation was reported between macular thickness and HbA1c values, but only in type 2 diabetes [22], while in this study only two retinal areas (peripheral and pericentral right areas) significantly correlate with metabolic parameters, HbAlc and microalbuminuria levels, respectively. In conclusion, our data confirm that duration of type 1 diabetes, lipid values, hypertension, microalbuminuria and HbAlc levels (considered both at the time of OCT evaluation and as mean values of the previous 5 years) are the most relevant factors for DR. This study suggests that there is no advantage in performing OCT routinely in patients with T1D without or with MDR in the absence of macular edema. Therefore, we conclude that screening of early DR should be performed by well-recognized conventional methods.

\section{References}

1. Giugliano D, Standl E, Vilsbøll T, Betteridge J, Bonadonna R, Campbell IW, Schernthaner GH, Staels B, Trichopoulou A, Farinaro E (2009) Is the current therapeutic armamentarium in diabetes enough to control the epidemic and its consequences? What are the current shortcomings? Acta Diabetol 46(3):173-181

2. Cerbone AM, Macarone-Palmieri N, Saldalamacchia G, Coppola A, Di Minno G, Rivellese AA (2009) Diabetes, vascular complications and antiplatelet therapy: open problems. Acta Diabetol 46(4):253-261

3. Pfister F, Wang Y, Schreiter K, Vom Hagen F, Altvater K, Hoffmann S, Deutsch U, Hammes HP, Feng Y (2010) Retinal overexpression of angiopoietin-2 mimics diabetic retinopathy and enhances vascular damages in hyperglycemia. Acta Diabetol 47(1):59-64

4. Djuric Z, Sharei V, Rudofsky G, Morcos M, Li H, Hammes HP, Nawroth PP, Bierhaus A, Humpert PM, Jonas JB (2010) Association of homozygous SDF-1 3'A genotype with proliferative diabetic retinopathy. Acta Diabetol 47(1):79-82

5. Moss SE, Klein R, Kessler SD, Richie KA (1985) Comparison between ophthalmoscopy and fundus photography in determining severity of diabetic retinopathy. Ophthalmology 92(1):62-67

6. McIntosh A, Peters J, O'Keeffe C, Khunti K, Baker R, Booth A (2000) Effectiveness of screening and monitoring tests for diabetic retinopathy a systematic review. Diabet Med 17(7):495-506

7. Lang GE (2007) Optical coherence tomography findings in diabetic retinopathy. Dev Ophthalmol 39:31-47

8. Baumal CR (1999) Clinical applications of optical coherence tomography. Curr Opin Ophthalmol 10:182-188

9. Schaudig UH, Glaefke C, Scholz F, Richard G (2000) Optical coherence tomography for retinal thickness measurement in diabetic patients without clinically significant macular edema. Ophthalmic Surg Lasers 31(3):182-186

10. Panozzo G, Gusson E, Parolini B, Mercanti A (2003) Role of OCT in the diagnosis and follow up of diabetic macular edema. Semin Ophthalmol 18(2):74-81

11. Alkuraya H, Kangave D, Abu El-Asrar AM (2005) The correlation between optical coherence tomographic features and severity of retinopathy, macular thickness and visual acuity in diabetic macular edema. Int Ophthalmol 26(3):93-99

12. Hee MR, Puliafito CA, Duker JS, Reichel E, Coker JG, Wilkins JR, Schuman JS, Swanson EA, Fujimoto JG (1998) Topography of diabetic macular edema with optical coherence tomography. Ophthalmology 105(2):360-370

13. Browning DJ, Fraser CM, Clark S (2008) The relationship of macular thickness to clinically graded diabetic retinopathy severity in eyes without clinically detected diabetic macular edema. Ophthalmology 115(3):533-539

14. Pires I, Bernardes RC, Lobo CL, Soares MA, Cunha-Vaz JG (2002) Retinal thickness in eyes with mild nonproliferative retinopathy in patients with Type 2 diabetes mellitus. Comparison of measurements obtained by retinal thickness analysis and optical coherence tomography. Arch Ophthalmol 120(10):1301-1306

15. Massin P, Erginay A, Haouchine B, Mehidi AB, Paques M, Gaudric A (2002) Retinal thickness in healthy and diabetic subjects measured using optical coherence tomography mapping software. Eur J Ophthalmol 12(2):102-108

16. Biallosterski C, Van Velthoven ME, Michels RP, Schlingemann RO, Devries JH, Verbraak FD (2007) Decreased optical coherence tomography-measured pericentral retinal thickness in patients with diabetes mellitus type 1 with minimal diabetic retinopathy. Br J Ophthalmol 91(9):1135-1138

17. Early Treatment Diabetic Retinopathy Study Research Group (1991) Grading diabetic retinopathy from stereoscopic color fundus photographs - an extension of the modified Airlie House classification. ETDRS Report Number 10. Ophthalmology 98:786-806

18. Early Treatment Diabetic Retinopathy Study Research Group (1985) Photocoagulation treatment for Diabetic Macular Edema. ETDRS Report Number 1. Arch Ophthalmol 103:1796-1806 
19. Goebel W, Kretzchmar-Gross T (2002) Retinal thickness in diabetic retinopathy - a study using optical coherence tomography. Retina 22:759-767

20. Lattanzio R, Brancato R, Pierro L, Bandello F, Iaccher B, Fiore T, Maestranzi G (2002) Macular thickness measured by optical coherence tomography (OCT) in diabetic patients. Eur J Ophthalmol 12(6):482-487

21. Bressler NM, Edwards AR, Antoszyk AN, Beck EW, Browning DJ, Ciardella AP, Danis RP, Elman MJ, Friedman SM, Glassman
AR, Gross JG, Li HK, Murtha TJ, Stone TW, Sun JK, Diabetic Retinopathy Clinical Research Network (2008) Retinal thickness on Stratus optical coherence tomography in people with diabetes and minimal or no diabetic retinopathy. Am J Ophthalmol 145(5): 894-901. Epub 2008 Feb 21

22. Chou TH, Wu PC, Kuo JZ, Lai CH, Kuo CN (2009) Relationship of diabetic macular oedema with glycosylated haemoglobin. Eye 23(6):1360-1363 